\title{
Safe sex: the train your mind (revise)
}

\author{
Rahul Hajare* \\ Department of Quality Assurance, Pune University, Pune, India
}

\begin{abstract}
The role of the mind in sex is hugely underestimated. To truly maximize sex, we not only have to be strategic about how we use our body, we also have to be strategic about how we use our mind. There are ways for our mind to communicate with our body to literally target sex. If we do not use our mind in this way, instead of sex we will just get unsafe sex and hormone destabilization. Furthermore, it is essential to target specific muscles through focus and awareness or else our sex will be $30 \%$ less effective. Think about it. By simply using our mind correctly, a sex-time would be just as effective as a safe sex time matrix. Diseases that could have been prevented by supplementing with minor changes in lifestyle to bring about years of vibrant health. Preventive health care for married couple never be ignored. Scientific research has been to consider the practice of and extent of safe sex that decontaminates the built environment and thus reduces the exposure of STD. What is "safe"? People tend to perceive "safeness" particularly in the built environment, and especially in their homes, society, from a visual perspective. Safe sex practices became more prominent in the late 1980s as a result of the AIDS epidemic. Promoting safer sex campion is now one of the aims of sex education. It is regarded as a harm reduction strategy aimed at reducing risks. Sex attraction is not accepted center pillars of love. Preventive health screening for married couple is simple and non-invasive. Over the years the causes of mortality and morbidity have changed from infective diseases like HIV, tuberculosis to chronic diseases. These diseases can be easily prevented if one understands the risk factors. If the risk factors are managed these diseases can be easily controlled. These include tests for general wellbeing and specific tests for STD. A complete blood count, along with immunity function profile and kidney function profile, is required to know how the various systems in the body work. Between the ages of 21 and 29 are the ages of aggression of sex. Between the ages of 30 and 50 is the post sex aggression period. The other most common sex education is difficult to identify due to hesitation. It is difficult to control due to very rare population size of people living with genital warts and they are not willingly enrolled to this study.
\end{abstract}

\section{Statement of purpose}

Safe sex practices became more prominent in the 21 st century as a result of the zero HIV new infection. Sex has much definition, safe sex accepted as a center pillar of love in married couple. Sex is not just penetrative vaginal sex. It can also include oral sex, anal sex and masturbation. Environment may even define sex as any sexual activity. When it comes to sex it is important that you make sure that the sex you have is always done safely. The risk reduction of safe sex is not absolute; for example, the reduced risk to the receptive partner of acquiring HIV from HIV-seropositive partners not wearing condoms compared to when they wear them is estimated to be about a four to fivefold. There has been much recent talk about how to target the rising of unsafe sex across the world, one of the biggest threats to global health today. While there is no doubting the size of the problem facing scientists, healthcare professionals and physicians, there are innovative ways we can target unsafe sex in the short term, which are discussed in three articles published in Essays in Biochemistry. False directs immunity born and accepted due to unsafe sex. There are different terminologies of sex; safe sex has one in a week with contraceptive. It is also a study of variation in differences dots of contraceptive and their application while having a sex. Safe sex it a sum of skin physiology can be reaches to build immunity across an environment [1]. India is second country in the world, who has addicted for porn and follower has increased day by day.

The best definition for safer sex is the statistic one in week - a reminder that beyond a point, one cannot control or ever completely prepare for the future. Believes unsafe sex afflicts those who have a sinful past, people cannot compensate for the sin against the unseen. But when you see the background, it will be found it was divine justice, nothing else. Sexual transmitted diseases mean no safe sex. After a year there has been pooling of common warts on face of men it is a sign of unsafe sex with age variation in sex partner. Unscientific opinion that illness is only two plus human to fall back on fantasy, or religion, when there is no rational explanation for random misfortunes. Sexrelated aggression has been accepted up to certain age and dignity also cultural draws has playing important role to educate about safe sex. A curved penis may be a sign of unsafe sex which has been warning early cause of cancer and simultaneously efflux of genital warts, some other types of common warts has polling skin [2]. Sex partner having age variation found the immunity converted into autoimmune system and that an end point of result of sexually transmitted disease. Safe sex with contraceptive can be avoiding visible warts using especially married couple. Using a contraceptive sex aggression towards another observer may be reduce. Today's market is full of various contraceptive, dotted express male contraceptive can reduce the chances of allergies reaction and sexually transfer diseases? Safe sex methodology will be changing after awareness about sex education. The leaching of warts is an example of autoimmunity which can be lead to common warts discharge among married couple and unmarried male female may be a critical determinant of immune dysfunction skin physiology. Safe sex and displacement of melanin secretion has quite close linkage between them. How melanin secretes, researchers have reported that non-teaching men staffs with these experiences are more difficult to treat; many do not access treatment and those who do, frequently do not stay because of difficulty maintaining helping relationships. Sex

${ }^{\star}$ Correspondence to: Rahul Hajare, Department of Quality Assurance, Pune University, Pune, India, Email: rahulhajare@rediffmail.com

Received: April 03, 2018; Accepted: April 25, 2018; Published: April 27, 2018 
aggression is directly proportional to physical maturity if maturity has beyond age unsafe sex desired simultaneously increases. Unsafe sex may increase proliferation rest of the capacity and body behaviour convert dysfunctioning of skin physiology. Aggression up to some level has really observed and safe sex can be handling with knowledge and curiosity and it is a source of evolution of micro tissues across the body function, soft tissue body mechanism is made by and restored by safe sex those having once a week [3].

\section{Conclusion}

Safe sex has sexual activity engaged in by people who have taken precautions to protect themselves against sexually transmitted infections (STIs) such as HIV. It has also referred to as safer sex or protected sex, while unsafe or unprotected sex has sexual activity engaged in without precautions. Sex aggression of human begins before 21. It is strongly recommended that women with an average risk of STD should undergo regular unsafe sex starting at the age 30 and shrink the vocal cord. Those who are between the age of 30 to 40 should be screened annually by sexologist. Men of 45 and older can have biennial screening or can continue screening annually. In addition to these, tests for skin physiology and Geriatric Dermatology are important. A clinical dermatology and a Mantoux skin test also needs to be done at least once in two years. These men women perspective has not been previously studied, particularly in this area of safe sex awareness. The purpose of this study is to describe the experience of seeking help for alcohol dependency by men women with and a history of chain alcohol dependency with other men the context in which it occurs.

\section{Acknowledgment}

This research work is in completed under the supervision and guidance Renowned Laboratory Scientist, Respected Dr. Ramesh S. Paranjape Retired Director \& Scientist 'G' National AIDS Research Institute, India. I express my sincere gratitude towards Respected Sir for motivation and being great knowledge source for this work.

\section{References}

1. Rahul Hajare (2018) "Safe Sex: The True Principal Health?". Medical Research and Clinical Case Reports 1: 79-81.

2. Rahul Hajare (2018) There is no Cure for the Cancer of Stupidity. Organic \& Medicinal Chem IJ 5: 555-655.

3. Kareem F Samhouri Health \& Hapiness.

Copyright: (C2018 Hajare R. This is an open-access article distributed under the terms of the Creative Commons Attribution License, which permits unrestricted use, distribution, and reproduction in any medium, provided the original author and source are credited. 